\title{
Le métadiscours du fantastique ou comment écrire après la théorie du genre
}

\author{
Clotilde Landais \\ Université de New South Wales
}

Le fantastique est un genre littéraire qui ne cesse de partager les spécialistes, ce qui fait dire à certains qu'il serait par nature indéfinissable. À l'inverse, faisant suite à Charles Nodier et à son manifeste "Du fantastique en littérature », paru en 1830, nombreux sont ceux qui ont tenté d'en donner une définition, créant une tradition de réflexion théorique dans laquelle les auteurs contemporains doivent trouver leur place. De façon consensuelle, serait fantastique tout récit de fiction ancré dans le quotidien de l'auteur et de son lecteur contemporain, mettant en scène des êtres ou des phénomènes échappant à toute explication rationnelle qui génèrent un sentiment d'angoisse 
chez les protagonistes et le lecteur. Si cela semble satisfaire la plupart des chercheurs, ceux-ci restent cependant divisés sur de nombreuses questions, comme les critères constitutifs du genre ou sa filiation.

Ainsi, pour certains chercheurs français comme Jean Fabre ou Louis Vax, le fantastique remonterait au XVIII ${ }^{\mathrm{e}}$ siècle, lorsque les Lumières érigèrent la raison en idéal philosophique. Selon ces chercheurs, ce mouvement avait pour chef de file l'écrivain allemand Ernst Theodor Amadeus Hoffmann (17761822 ) et se serait ensuite élargi à l'Europe artistique. Pour nombre d'auteurs et chercheurs anglo-saxons, à l'instar de Howard Philip Lovecraft ou de Peter Penzoldt, le fantastique ou ses avatars que sont les mythes, légendes et autres contes merveilleux - existerait depuis toujours et dans toutes les cultures. Dès 1764, des auteurs anglais comme Horace Walpole, Anne Radcliffe et Matthew Gregory Lewis donnent naissance au roman gothique, qu'ils considèrent comme une branche du fantastique. Ce genre puise dans les sources folkloriques et mythologiques et a pour lieux de prédilection les châteaux, couvents et autres espaces rappelant le Moyen Âge. L'atmosphère y est souvent mélancolique et la peur ou la terreur n'y sont pas étrangères. Le chercheur français Denis Mellier tente quant à lui une synthèse, à laquelle nous souscrivons, de ces deux filiations possibles en soutenant que le roman gothique permit «à l'irrationnel de trouver une expression esthétique séduisante au cœur du siècle de la raison » et développa également chez les lecteurs " un goût pour des apparitions horrifiantes et des mises en scènes paroxystiques » (p. 18-19). 
Cette perspective théorique intégrative de Mellier trouve en fait naissance dans la pratique du fantastique horrifique contemporain. Nous allons nous intéresser ici à deux auteurs nord-américains qui, en tant que praticiens du genre, mais également en tant qu'enseignants, ont inséré dans leurs œuvres ces différents débats théoriques sur le fantastique. Nous prendrons comme objets d'étude le roman The Dark Half (1989) de l'États-Unien Stephen King et le roman Sur le seuil (1998) du Québécois Patrick Senécal. Nous étudierons successivement les métadiscours du fantastique et de l'écriture de l'horreur qui tiennent une place importante dans ces romans et reflètent la façon dont King et Senécal articulent dimension théorique et écriture dans leur œuvre.

\section{Les métadiscours du fantastique}

Comme nous l'avons mentionné, un courant du fantastique serait né au XVIII ${ }^{\text {e }}$ siècle en réaction à la raison, elle-même érigée en idéal philosophique par les Lumières contre les superstitions religieuses. Or, comme le dit Vax,

[u]n peu de savoir éloigne de la superstition, beaucoup de savoir y ramène : devise de la littérature fantastique comme de l'obscurantisme. [...] C'est parce que les événements étaient réputés incroyables hier qu'ils apparaissent fantastiques aujourd'hui. (p. 60)

Cette remarque trouve un écho dans Sur le seuil de Senécal, où le prêtre tient un discours qui renvoie aux origines du genre:

C'est un peu ironique comme situation, non? La religion et la science qui se consultent... Il y a cinquante ans, quand les gens voulaient connaître la vérité, ils se tournaient vers la religion. Puis, peu à peu, par frustration, ils se sont tournés vers la 
science. Et nous voilà, l'un en face de l'autre... sans réponse claire. (p. 353)

Bien que ce métadiscours se situe temporellement dans le contexte québécois, il rappelle que le fantastique, du point de vue d'un courant théorique littéraire, est né tant du rejet des superstitions religieuses que de celui du rationalisme. En effet, comme le souligne le prêtre du roman, ni la religion ni la science ne peuvent apporter de réponses à certains phénomènes. Seul le fantastique le peut.

Or, il est intéressant de constater que, malgré la présence de ce métadiscours sur le genre dans le roman québécois, le terme «fantastique » n'est jamais employé. Les personnages et le narrateur homodiégétique en particulier, psychiatre préfèrent parler de l'« irrationnel ${ }^{1} »$, se plaçant ainsi directement face à la raison :

Je doute de l'ordre habituel des choses. Je doute de la toutepuissance de la logique. Je me dis que peut-être... peut-être que le cas Roy relève d'une explication... non rationnelle. [...] Je dis bien peut-être, Jeanne. Ça veut dire que je vais continuer à chercher une explication dans le cas de Roy, mais en me disant que cette explication peut être autant irrationnelle... que rationnelle... (p. 258)

Le narrateur psychiatre de Sur le seuil tout comme le shérif dans The Dark Half apparaissent comme les parangons du rationalisme. De par leur profession, tous deux s'inscrivent dans la tradition littéraire fantastique du narrateur ou de l’observateur «Gardien de la Raison » (Senécal, 1998, p. 168), rôle généralement tenu par un savant, un médecin, un

\footnotetext{
1 Senécal s'oppose par là à Bessière (1974), pour qui «[i]l ne faut pas assimiler fantastique et irrationnel. De Cazotte à Lovecraft, le récit fantastique est celui de l'ordre qui ne décrit point l'illégal pour récuser la norme, mais pour la confirmer » (p. 28).
} 
enquêteur, un enseignant ou un écrivain. À ce titre, si l'écrivain fictif de Sur le seuil semble ne jamais comprendre la nature du phénomène qui le possède, l'attitude de celui de The Dark Half est plus surprenante:

Reason and rationality told him that Stark could not be out there, ramming around like some weird cancer in human form, killing people. [...]

He was, though. Thad knew he was $[\ldots]^{2}$. (p. 164)

Bien que sa position d'écrivain eût dû le placer également comme défenseur de la raison, Beaumont semble accepter le fait que son pseudonyme ait pris vie. Ainsi, le rôle d'esprit rationnel revient uniquement au shérif, l'enquêteur :

"Are you done?"

She looked at Alan, startled, as if his voice had brought her out of a deep private reverie. "What?"

"I asked if you were done. You wanted to have your say, and I want to make sure you got it."

Her calm broke. She fetched a deep sigh and ran her hands distractedly through her hair. "You don't believe it, do you? Not a single word of it ${ }^{3}$.” (p. 204)

Dans Sur le seuil, ce rôle revient plus traditionnellement au narrateur - psychiatre mais également enquêteur (p. 298) -

${ }^{2}$ «Tout son côté rationnel lui disait qu'il était impossible que Stark soit là-dehors, dans la nature, rôdant comme quelque cancer dément à forme humaine et tuant des gens. [...] Et pourtant, Thad savait, et Liz savait aussi, que c'était [le cas] » (King, 1990, p. 169).

3 "“Terminé?" / Liz regarda Alan, surprise, comme si sa voix la tirait d'une profonde rêverie intime dans laquelle elle aurait été plongée. / "Quoi?" / "Je demande si vous en avez terminé. Vous vouliez pouvoir vous exprimer, et je veux être sûr que vous l'avez fait." / Sur-le-champ, elle perdit sa belle façade de calme. Elle poussa un profond soupir et passa une main distraite dans ses cheveux. "Vous ne croyez pas un traitre mot de ce que nous avons dit, c'est ça?” » (King, 1990, p. 208). 
qui va refuser aussi longtemps que possible l'explication surnaturelle aux événements dont il est témoin, car son « travail ne [1]'autorise pas à croire à de telles choses » (p. 351):

J'ai compris, j'ai compris, bordel! ai-je envie de lui hurler. J'ai compris ce que tu veux dire! Comme j'ai compris ce que Monette a essayé d'insinuer! Comme j'ai compris les doutes de Jeanne cet après-midi! Je vous ai tous compris! Mais ça se peut pas, comprenez-vous ça? ça se peut pas! (p. 202)

La présence d'enquêteurs comme narrateurs ou témoins des événements fantastiques reprend la proposition d'Irène Bessière, qui «li[e] le fantastique à une enquête, conduite d'un point de vue rationaliste, sur les formes de la rationalité » (p. 59). Jean Fabre fait quant à lui remonter l'origine du roman policier à ce qu'il appelle les « contes de ratiocination de Poe [qui] semblent manifestement issus du Fantastique expliqué d'Ann Radcliffe» (p. 59). Ainsi, « les deux formules romanesques jouent [...] du même code du vraisemblable générique : la solution la plus invraisemblable au départ sera toujours la bonne [même si] cet invraisemblable ne sera pas le même [entre le fantastique et le policier] » (Fabre, p. 160). King établit notamment ce rapport entre roman policier et texte fantastique en inscrivant les romans du pseudonyme dans The Dark Half dans le genre thriller, ce qui entraîne un métadiscours sur le genre de la part de l'écrivain fictif:

Well, Stark messed with some of the conventions of the mystery story. Never anything so Agatha Christie as the scenario I suggested, but that doesn't mean I can't think that way if I put my mind to it ${ }^{4}$. (p. 105)

\footnotetext{
4 « "Eh bien, voyez-vous, Stark s'en prend à quelques-unes des conventions du roman noir. Rien [d'aussi] agatha-christien que le scénario que je vous ai
} 
Comme nous l'avons souligné, Sur le seuil est également bâti comme une enquête. Cette construction est marquée par de nombreuses références au roman ou au film policiers. Ainsi, l'attitude du journaliste est fréquemment renvoyée à celle des héros de romans ou de films policiers : «Il adopte un petit air "mystérieux-de-film-policier" et je ne peux m'empêcher de le trouver totalement grotesque » (p. 35). Ou encore : «Il se croit dans un film policier, ma parole! » (p. 87). Cependant, l'attitude dédaigneuse du narrateur change à cet égard lorsque lui-même se laisse prendre au jeu de l'enquête, se comparant par exemple à Sherlock Holmes : " Roy relève la tête avec surprise. Il doit se demander comment nous savons ça. Je ressens une pointe de fierté enfantine. Sherlock Lacasse vient de marquer un point. » (p. 212) Ainsi, pris entre son esprit rationnel et les événements surnaturels, le narrateur est conduit à une contradiction :

"On va trop vite, Jeanne. Avant d'aborder des hypothèses irrationnelles, il faut trouver des preuves tangibles..."

"Des preuves de l'irrationnel... Un peu contradictoire, non?" (p. 269)

Ces références, qui brisent l'illusion référentielle, tiennent également lieu de métadiscours sur la construction du récit fantastique. En rappelant les mécanismes du genre, les personnages rendent le processus d'écriture visible. Par ailleurs, comme le remarque Linda Hutcheon, « [t]he [...] use of detective plots [...] conventions points to the importance of the event of reading as having a role in literary creation, a role as

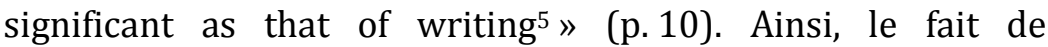

suggéré, mais ça ne signifie pas que je ne suis pas capable de vous en sortir un, si je m'y mets" » (King, 1990, p. 110).

5 Je traduis: "Le recours aux conventions du roman policier indique que l'acte de lecture tient un rôle important dans la création littéraire et que ce rôle est tout aussi significatif que celui d'écriture. » 
représenter un protagoniste écrivain dans un récit fantastique sous forme d'enquête permet de souligner l'importance du rôle du lecteur, chargé de décrypter les métadiscours sur le genre. Fabre le souligne:

[C]ette lucidité de la lecture policière ou plutôt ratiocinante abhorre et détruit la participation aliénée du "Fantastique » [...]. Le fantastique n'est pas une question de croyance réelle et de sincérité mais de savoir-faire et savoir-écrire [...]. (p. 165)

C'est bien parce que le fantastique est un « savoir-écrire » que la réflexion menée par Stephen King et Patrick Senécal sur le genre aurait été incomplète sans un métadiscours sur l'écriture de l'horreur telle que définie par Noel Carroll :

[A] narrative [...] in which at least one monster appears, such as that the monster in question is designed to elicit an emotional response from us that is a complex compound of fear and disgust in virtue of the potential danger or threat the monster evinces and in virtue of its impurity ${ }^{6}$. (p. 151)

\section{Les métadiscours sur l'écriture de l'horreur}

Le premier métadiscours sur l'écriture de l'horreur présent dans Sur le seuil et The Dark Half concerne la fascination pour l'horreur. Ainsi, le narrateur du roman québécois s'interroge sur l'engouement du public - y compris celui de sa collègue pour l'horreur. Jeanne présente en effet ainsi les romans de Roy :

\footnotetext{
${ }^{6}$ Je traduis : «Tout récit [...] dans lequel un monstre au moins apparaît, à condition que ce monstre soit conçu pour susciter en nous une émotion. Cette émotion est un mélange complexe de peur et de dégoût en vertu du danger potentiel ou de la menace que le monstre incarne, et en vertu de son impureté. »
} 
"[...] il a une façon unique de décrire l'horreur! [...] Il a le tour de nous faire entrer dans des choses insoutenables... Ses descriptions sont tellement détaillées... Et l'ambiance, Paul, l'ambiance de ses histoires... [...] Je n'ai jamais rien lu de pareil."

Je me contente de hocher la tête; vaguement déconcerté. Étonnant, quand même, l'engouement que les gens ressentent pour l'horreur! Comment peut-on avoir envie de lire un roman qui déclenche un sentiment qu'on devrait, de prime abord, vouloir éviter? Pourtant, les faits sont là : Thomas Roy vend des millions de livres dans le monde. Cela m'échappe complètement. (p. 19-20)

En soulignant les raisons de l'efficacité des romans de Roy, le personnage tient un métadiscours sur les règles à respecter pour écrire un bon roman d'horreur. Quant aux interrogations $\mathrm{du}$ narrateur, elles renvoient le lecteur à ses propres motivations alors même qu'il est précisément en train de lire un roman d'horreur.

Si le roman ne fournit pas de réponses à cette fascination qu'exerce l'horreur, le narrateur explicite l'origine de son sentiment de rejet :

Quand on a côtoyé de tels patients pendant un certain temps, nos réactions face à eux deviennent complexes. Nous ne pouvons plus nous contenter de ressentir tout simplement du dégoût ou de la haine, comme tout le monde. Il y a aussi de la curiosité et de la fascination. C'est cela qui m'écœure le plus. L'horreur fascine. Et je ne veux plus être fasciné par ce sentiment. Je l'ai compris quand Boisvert a vidé le contenu de ses yeux sur mon visage. (p. 146)

Le narrateur semble signaler ici que l'on reste fasciné par l'horreur jusqu'à ce qu'on la vive. Par ailleurs, il dresse de façon implicite un parallèle entre les psychiatres et les écrivains de littérature d'horreur qui s'interrogent sans relâche sur cette fascination. Cependant, à l'occasion du récit enchâssé dans 
lequel un jeune punk raconte comment l'écrivain fictif a perdu son œil, le narrateur découvre que la fascination pour un récit d'horreur est différente de celle ressentie par un psychiatre dans l'exercice de ses fonctions:

Je crois que j'aurais donné deux fois le même montant pour entendre la suite. Pour la première fois, je commence à comprendre la fascination que peuvent ressentir les lecteurs de Roy pour l'horreur [...]. (p. 235)

Ainsi, si le narrateur ne veut plus être fasciné par l'horreur des psychopathes - ce que Noel Carroll appelle la «real-life horror $^{7} »$ (p. 147) —, il réalise que la fascination ressentie à la lecture - ou, comme ici, à l'écoute - d'un récit d'horreur est différente. En reconnaissant son impatience à connaître la suite de l'histoire, le narrateur légitime les lecteurs - et, par là même, les auteurs - de littérature d'horreur que Carroll appelle par distinction la « art-horror ${ }^{8}$ » (p. 147). Le lecteur de Sur le seuil, bien que ramené par ce métadiscours à la fictionnalité du récit, se trouve par conséquent «autorisé » à poursuivre son acte de lecture.

Le deuxième métadiscours sur l'écriture de l'horreur concerne le rapprochement avec l'humour, présent dans les deux romans :

[Thad] knew that humor rarely if ever had anything to do with such fits. In fact, the cause was apt to be something quite the opposite of funny.

Something to be afraid of, maybe9. (King, 1989, p. 21)

\footnotetext{
7 Je traduis : « l'horreur de la vie réelle ».

8 Je traduis : «l'horreur artistique ».

9 « [Thad] savait que l'humour avait rarement à voir avec de tels accès [de fou rire] - à vrai dire, à peu près jamais. La cause, à vrai dire, avait toutes les
} 
Je hausse les sourcils, soudain pris d'une furieuse envie de rire. Dans un autre contexte, cela aurait pu ressembler à un gag. J'avais déjà entendu quelque part que l'horreur et l'humour sont deux émotions très semblables. Ce n'est peut-être pas si faux. (Senécal, 1998, p. 120)

Ces deux extraits renvoient presque explicitement à l'article de Noel Carroll, «Horror and Humor », où il est montré comment un même stimulus, en fonction du contexte, peut générer deux réponses émotionnelles généralement qualifiées de différentes (p. 147) :

In order to transform horror into laughter, the fearsomeness of the monster - its threat to human life - must be sublated or hidden from our attention. Then we will laugh where we would otherwise scream ${ }^{10}$. (p. 158)

Ainsi, en supprimant l'élément effrayant, le monstre peut devenir comique. Une fois encore, le métadiscours dévoile dans le corps du roman le mécanisme d'écriture de l'horreur.

Enfin, le troisième métadiscours porte sur le rapport à la réalité dans la littérature d'horreur. Dans Sur le seuil, par le biais du personnage de l'agent littéraire, l'inspiration réaliste est justifiée par une référence aux auteurs désormais classiques du XIXe siècle :

"C'est un peu sans-cœur, hein, Pat? S'inspirer des vraies horreurs pour écrire des histoires à sensations fortes!" Làdessus, je m'étais empressé de le rassurer! Tous les écrivains faisaient ça! Hugo, Zola, Balzac... Ils se sont tous inspirés de la réalité pour écrire leurs chefs-d'œuvre! (p. 119)

chances d'être le contraire de comique. / À chercher plutôt du côté de ce qui faisait peur, peut-être » (King, 1990, p. 31).

10 Je traduis : «Pour transformer l'horreur en rire, notre attention doit être distraite ou détournée du caractère effrayant du monstre, c'est-à-dire de la menace qu'il représente pour la vie humaine. Alors nous pourrons rire où nous aurions dû hurler. » 
Un acteur de l'institution littéraire tient un discours visant à établir le rapport entre la littérature d'horreur et le courant réaliste. Cependant, par le biais du narrateur homodiégétique, ce métadiscours est immédiatement contredit par l'argument généralement tenu par les détracteurs du genre : «Comparer la littérature sanglante et populaire de Roy aux romans sociaux et réalistes de Balzac me semble d'un goût douteux [...]. » (p. 120) Ainsi ces deux métadiscours tendent-ils à briser l'illusion référentielle et à renvoyer le lecteur aux controverses portant sur la qualité littéraire du genre.

De même, certaines remarques soulignent la construction du roman québécois, pointant par exemple le caractère improbable, irréaliste, de certains raisonnements favorables au surnaturel : " "Ça ne tient pas debout, Jeanne! Tu entends ce que tu racontes? C'est fou! Même les Américains n'oseraient mettre une telle scène dans un film!” » (p. 167). Cependant, par contraste, ce métadiscours rationaliste indique au lecteur que, puisqu'il s'agit d'un roman fantastique, l'auteur ira au-delà de ce que le narrateur du récit n'ose imaginer. Parallèlement - et toujours en opposition au cinéma états-unien -, un autre métadiscours sur le réalisme en fiction souligne le rejet du happy ending: " “On n'est pas dans un film américain où les simples vertus du héros lui permettent de se reprendre en main... La réalité est plus ingrate.” »(p. 261) Le narrateur procède ainsi à une prolepse indiquant au lecteur que le réalisme dans lequel est ancré tout récit fantastique interdit les fins heureuses et que le roman qu'il lit ne fera pas exception à la règle. 
Dans The Dark Half, à la différence du roman québécois, ce métadiscours sur le réalisme porte sur la réalité de l'univers imaginaire pour l'écrivain de fiction :

"[Stark] seemed real to me when I was writing as him, but only in the way all the stories I write seem real to me when I'm writing them. Which is to say, I take them seriously but I don't believe them... except I do... then...11" (p. 206)

L'écrivain fictif justifie ainsi son acceptation quasi immédiate de l'incarnation de son pseudonyme, puisqu'il le considérait déjà comme une personne réelle. Poe notait déjà cette emprise de l'imagination : "So entire is my faith in the power of words, that at times I have believed it possible to embody even the evanescence of fancies such as I have attempted to describe ${ }^{12}$ » (p. 116-118). L'écrivain fictif de The Dark Half reconnaît cependant ne jamais en avoir parlé auparavant et le regrette dans la mesure où cela empêche le shérif de comprendre - et d'accepter - les explications de l'écrivain quant à l'existence de son pseudonyme:

"All the times I've talked about writing," he said. "Hundreds of lectures, thousands of classes, and I don't believe I ever said a single word about fiction-writer's grasp of the twin realities that exist for him - the one in the real world and the one in the manuscript world. I don't think I ever even thought about it.

\footnotetext{
11 « "[Stark] me paraissait réel quand j'écrivais sous son nom, mais seulement de la manière dont toutes les histoires que je raconte me semblent réelles pendant que je les écris. Autrement dit, je les prends au sérieux, mais je ne les crois pas... sauf qu'au fond... alors..." » (King, 1990, p. 209)

12 « [M]a foi dans le pouvoir des mots est si entière que, certains jours, j'ai cru possible de fixer jusqu'à l'évanescence de ces intuitions que je viens d'essayer de décrire. » (Poe, 1983, p. 99)
} 
And now I realize... well... I don't even seem to know how to think about it ${ }^{13}$." (p. 206)

Par le biais de ce métadiscours tenu par l'écrivain fictif, King annonce son désir d'expliquer les deux réalités qu'il perçoit en tant qu'auteur de fiction. Ce métadiscours aboutit en octobre 2006 à la publication de Lisey's Story, autre roman fantastique sur l'écriture, où ce rapport entre réel et imaginaire est développé.

L'exemple de Lisey's Story, publié plus de quinze ans après The Dark Half, souligne bien la nécessité pour certains auteurs contemporains d'inclure une réflexion théorique dans leur écriture. Senécal n'est pas en reste, comme le montre, par exemple, la publication, en 2000, de son roman Aliss, dans lequel le métadiscours du conte, allié à une lecture structuraliste et hypertextuelle, permet à l'auteur québécois de donner son analyse des contes de Lewis Carroll (voir Landais, 2009). Ainsi, la tradition de réflexion sur le genre fantastique commencée avec Nodier au XIX ${ }^{\mathrm{e}}$ siècle existe toujours chez les auteurs contemporains. Loin de limiter la créativité artistique, l'inscription de métadiscours sur le fantastique ou l'écriture de l'horreur contribue au contraire à son enrichissement. En s'appuyant à la fois sur les courants du fantastique intérieur d'Hoffmann et sur ceux du roman gothique de Walpole ou

\footnotetext{
13 «"Quand je pense au nombre de fois où j'ai parlé de ce que c'était, l'écriture", reprit-il. "Des centaines de conférences, des milliers d'heures de cours, et je ne crois pas avoir jamais prononcé un seul mot sur la manière dont l'auteur de fiction appréhendait la réalité jumelle qui existe pour lui celle du monde véritable et celle du monde de son manuscrit. Et je me rends compte maintenant... eh bien... il ne me semble même pas savoir comment la penser". » (King, 1990, p. 110)
} 
Radcliffe, Stephen King et Patrick Senécal mettent en pratique dans leur écriture la perspective intégrative sur le genre théorisée par Denis Mellier. En faisant tenir à leurs personnages des propos inspirés par les chercheurs, en soulignant les mécanismes du genre, les auteurs rendent le processus d'écriture visible. Si de tels métadiscours brisent l'illusion référentielle, ils mettent en revanche en avant le jeu cognitif qui se tient depuis toujours en littérature de genre ${ }^{14}$ entre l'auteur et le lecteur (voir Bleton, 1999). Au lieu de nier ses influences, l'auteur de fantastique les assume, générant par là même un dialogue intertextuel avec le lecteur coopérant (voir Eco, 1985, p. 65). Les œuvres fantastiques de King et de Senécal sont donc fortement empreintes de cette dimension métatextuelle soidisant propre à la littérature postmoderne, ce qui rappelle la pertinence des interrogations posées par Harold Bloom sur la gestion des influences littéraires, mais également théoriques, dans le processus créatif.

\section{Bibliographie}

BESSIÈRE, Irène. (1974), Le Récit fantastique: la poétique de l'incertain, Paris, Larousse.

Bleton, Paul. (1999), Ça se lit comme un roman policier... comprendre la lecture sérielle, Québec, Nota bene, coll. «Études culturelles ».

\footnotetext{
14 À l'instar de chercheurs comme Anne Besson, j'appelle «littérature de genre» ce qui est parfois qualifié de «littérature populaire», de « paralittérature » voire de « sous-littérature » et qui recouvre principalement les littératures de l'imaginaire (fantastique, fantasy et science-fiction) et le roman policier.
} 
Carroll, Noel. (1999), "Horror and Humor », The Journal of Aesthetics and Art Criticism, Vol. 57, No. 2, p. 145-160.

Eco, Umberto. (1985 [1979]), Lector in fabula : le rôle du lecteur, Paris, Grasset.

FABRE, Jean. (1992), Le miroir de sorcière : essai sur la littérature fantastique, Paris, José Corti.

HUTCHEON, Linda. (1987), « Metafictional Implications for Novelistic Reference », in Anna Whiteside \& Michael Issacharoff (eds.), On Reference in Literature, Indianapolis, Indiana University Press.

KING, Stephen. (1989), The Dark Half, New York, Signet.

—. (1990), La Part des ténèbres, Paris, Albin Michel.

—. (2006), Lisey's Story, New York, Scribner.

LANDAIS, Clotilde. (2009), "Aliss de Patrick Senécal comme hypertexte des contes de Lewis Carroll », Clair-Obscur, vol. 5, p. 50-61.

MELLIER, Denis. (2000), La Littérature fantastique, Paris, Seuil, coll. « Mémo ».

NoDIER, Charles (1961 [1830]), « Du fantastique en littérature », dans Contes, Paris, Garnier-Frères.

PoE, Edgar Allan Poe. (1846), «Marginalia », Graham's Magazine, XXVIII, March 1846, p.116-118, sur http://books.eserver.org/fiction/poe/marginalia.html

—. (1983), Préfaces et marginalia, Aix-en-Provence, Alinea.

SENÉCAL, Patrick. (1998), Sur le seuil, Beauport, Alire.

-. (2000), Aliss, Beauport, Alire. 
VAX, Louis. (1987), La Séduction de l'étrange, Paris, PUF, coll. «Quadrige ».

\title{
Résumé
}

La littérature fantastique ne cesse de partager les spécialistes, créant une tradition de réflexion théorique dans laquelle les auteurs contemporains doivent trouver leur place. Nous nous intéressons dans cet article à deux auteurs nord-américains de fantastique horrifique qui ont inséré ces différents débats théoriques dans leur œuvre. L'étude des romans The Dark Half de Stephen King et Sur le seuil de Patrick Senécal nous permet de voir comment les métadiscours du fantastique et de l'écriture de l'horreur rappellent la pertinence des interrogations posées par Harold Bloom sur la gestion des influences littéraires et théoriques dans le processus créatif.

\begin{abstract}
Horror fiction has always divided scholars, creating a tradition of theoretical thinking in which contemporary authors have to find their place. In this paper, I will examine two horror novels by North American authors who inserted these different debates in their works. The study of Stephen King's Dark Half and Patrick Senécal's Sur le seuil will allow me to show how the metadiscourse of horror fiction and the metadiscourse of horror writing underline the accuracy of Harold Bloom's questions on the management of literary and theoretical influences in the creative process.
\end{abstract}

